\title{
Ensino em saúde mental em instituições públicas de ensino superior de Alagoas: perspectivas político-pedagógicas dos docentes
}

\author{
Teaching mental health in public institutions of higher education in Alagoas: political- \\ pedagogical perspectives of teachers
}
Enseñanza en salud mental en instituciones públicas de enseñanza superior de Alagoas: perspectivas político-pedagógicas de los docentes

Ewerton Cardoso Matias ${ }^{1 *}$, Jorge Luís de Souza Riscado², Jarbas Ribeiro de Oliveira ${ }^{3}$.

\begin{abstract}
RESUMO
Objetivo: Analisar os saberes e práticas de ensino-aprendizagem do ensino em saúde mental em duas instituições públicas de ensino superior de Alagoas, bem como identificar as ferramentas e metodologias que têm sido utilizadas pelos docentes. Métodos: Estudo exploratório, transversal, numa perspectiva qualitativa, tendo por objeto de estudo à docência em saúde mental, representada por docentes de cinco cursos de graduação das duas instituições de ensino. Os dados foram produzidos a partir de entrevistas, utilizando-se um questionário estruturado e um roteiro de entrevista semiestruturado. O estudo foi aprovado por Comitê de Ética em Pesquisa. Resultados: Os resultados apontam que há variedade de ferramentas, estratégias didáticas, tais como rodas de conversa, explanação dialogada, seminários e debates, assim como cenários para a articulação teórico-prática utilizados pelos docentes e que os conteúdos e referenciais teóricos utilizados têm orientações distintas. Conclusão: Nessas instituições, o ensino tem sido pautado por estratégias que buscam formar o discente na perspectiva da Política Pública de Saúde Mental.
\end{abstract}

Palavras-chave: Ensino, Saúde mental, Política de saúde.

\begin{abstract}
Objective: To analyze the knowledge and practices of teaching-learning of teaching in mental health in two public institutions of higher education in Alagoas, as well as to identify the tools and methodologies that have been used by teachers. Methods: Exploratory, cross-sectional study, in a qualitative perspective, with the object of study being teaching in mental health, represented by teachers from five undergraduate courses at both educational institutions. The data were obtained from the choice, using a structured questionnaire and a semi-structured interview script. The study was approved by the Research Ethics Committee. Results: The results show that there are a variety of tools, didactics, such as conversation circles, dialogical explanation, seminars and debates, as well as scenarios for the theoretical-practical articulation used by the teachers and that the contents and theoretical references used have different orientations. Conclusion: In these institutions, teaching has been guided by the strategy that students seek from the perspective of Public Policy on Mental Health.
\end{abstract}

Keywords: Teaching, Mental health, Health policy.

RESUMEN

Objetivo: Analizar los conocimientos y prácticas de enseñanza-aprendizaje de la docencia en salud mental en dos instituciones públicas de educación superior en Alagoas, así como identificar las herramientas y metodologías que han sido utilizadas por los docentes. Métodos: Estudio exploratorio, transversal, en perspectiva cualitativa, con el objeto de estudio de la docencia en salud mental, representado por docentes de cinco carreras de grado de ambas instituciones educativas. Los datos se obtuvieron de la elección, mediante un cuestionario estructurado y un guión de entrevista semiestructurado. El estudio fue aprobado por

1 Universidade de Ciências da Saúde de Alagoas (UNCISAL), Maceió - AL.*E-mail: ewerton_to@hotmail.com

2 Universidade Federal de Alagoas (UFAL), Maceió - AL.

3 Universidade Federal de Alagoas (UFAL), Arapiraca - AL. 
el Comité de Ética en Investigación. Resultados: Los resultados muestran que hay variedad de herramientas, didácticas, como círculos de conversación, explicación dialógica, seminarios y debates, así como escenarios para la articulación teórico-práctica que utilizan los docentes y que los contenidos y referencias teóricas utilizados tienen diferentes orientaciones. Conclusión: En estas instituciones, la docencia se ha guiado por la estrategia que los estudiantes buscan desde la perspectiva de las Políticas Públicas en Salud Mental.

Palabras clave: Enseñanza, Salud mental, Política de salud.

\section{INTRODUÇÃO}

Durante a história da humanidade, percebe-se uma preocupação constante com a formação profissional daqueles que cuidam dos aspectos de saúde da população. Botti SHO (2009) diz que, desde épocas remotas, os candidatos a pajé em tribos indígenas, eram selecionados e treinados para exercerem a função. Conforme esse sistema, os discípulos aprendiam as habilidades na relação com os mestres, figuras de referência.

Pensando os fundamentos para a prática docente, Brito Filho GT, et al. (2004) destacam que o ato de aprender e ensinar constituem atividades muito próximas da experiência humana. Isso nos ajuda a refletir que, desde o nascimento, o homem vive a experiência da sobrevivência e também do desenvolvimento, o qual é alcançado pela aprendizagem, em meio à comunidade, que é renovada constantemente.

Em se tratando da docência no campo da saúde, Batista NA e Batista SH (2004) asseguram que é preciso agregar uma triangulação entre ensino-aprendizagem-assistência, e que, dessa forma, a competência profissional de cada área ganha destaque, a partir da inserção nos cenários de ensino e dos atores envolvidos nesse contexto, tais como o docente, o discente, usuários e a própria comunidade.

Em um estudo, Ristow AM (2007) diz que os avanços científicos e tecnológicos proporcionados pela ciência, numa racionalidade instrumental, em muito contribuíram para se distanciar de uma visão holística, em favor de uma medicina organizada por compartimentos, segmentada, tendo como foco a doença e não os sujeitos.

Para Yasui S (2010) muitos movimentos sociais surgidos na década de 1970, nascem a partir das necessidades cotidianas, como transporte, moradia e saúde, pois possibilitaram o reconhecimento de interesses sociais comuns e que fez emergir novos sujeitos políticos, os quais foram essenciais no processo de (re)construção da cidadania.

Por seu turno, o ensino para a área de saúde mental tem exigido um alinhamento maior do trabalho docente à política de saúde vigente, apesar de existirem formas diferentes de analisar o cenário posto. Em meio à ressignificação da lógica do cuidado pautado na exclusão, para uma lógica de promoção da saúde, cidadania, contratualidade social e na clínica ampliada, emerge a necessidade da produção de novos conhecimentos e com a diversidade de serviços que compõem a Rede de Atenção Psicossocial (RAPS), esse novo modus operandi tem exigido novas reflexões e formações sobre o cuidado (ALBUQUERQUE VS e CAMPOS JCL, 2010; AMARANTE P, 2007).

As Diretrizes Curriculares Nacionais (DCN) para os cursos de saúde, instituídas a partir de 2001, através da Lei 10.172 que aprova o Plano Nacional de Educação (BRASIL, 2001a) e parecer do Conselho Nacional de Educação (CNS) no 1.133/2001 (BRASIL, 2001b), definiram os princípios na formação de graduação para área da saúde. Na prática, essas Diretrizes orientam a educação em sintonia com o modelo de saúde vigente, - Sistema Único de Saúde (SUS), para responder às necessidades sociais de saúde na perspectiva da integralidade e da atuação em equipe. Essas características buscam repensar o processo de ensinoaprendizagem (CARNEIRO LA e PORTO CC, 2014).

Apesar de haver fragilidades indicadas na ampliação e efetivação do cuidado corresponsabilizado e contra-hegemônico, Ribeiro MC (2014) aponta alguns caminhos para superar as diferenças existentes entre o fazer nos serviços e o saber das universidades, sugerindo que aconteçam parcerias entres esses dois cenários para a efetivação do novo modelo de cuidado.

Em virtude do que vem sendo discutido, entre profissionais, a Política Nacional de Saúde e a atual conjuntura da atenção em Saúde Mental, e o ensino na área, buscou-se nesse estudo, analisar os saberes e 
práticas de ensino-aprendizagem do ensino em saúde mental em duas instituições públicas de ensino superior de Alagoas, bem como identificar as ferramentas e metodologias que têm sido utilizadas pelos docentes em suas práticas.

\section{MÉTODOS}

Estudo exploratório, transversal, qualitativo, tendo como objeto de estudo a docência em saúde mental, representada por docentes dos cursos de Enfermagem, Medicina, Psicologia, Serviço Social e Terapia Ocupacional e suas representações frente às práticas de ensino.

A amostra do estudo é não probabilística, por conveniência com 11 docentes, que foram convidados a participar desse estudo, a partir de suas inserções nas disciplinas/módulos nos cinco cursos de graduação, das universidades públicas de Alagoas. Os critérios de inclusão utilizados foram: Ser docente da área de saúde mental de uma das universidades; docente de disciplina ou módulo que trate de saúde mental em um dos cursos.

Foram realizadas entrevistas individuais nos locais de trabalho, entre os meses de julho/2015 a fevereiro/2016, utilizando-se um questionário e um roteiro semiestruturado buscando (1) perfil dos sujeitos e as (2) ferramentas utilizadas no processo de ensino-aprendizagem. Além de um quesito no qual o docente discorreu sobre o momento atual do ensino em saúde mental.

As entrevistas foram gravadas e transcritas em sua totalidade e seu conteúdo analisado à luz da Análise de Conteúdo Bardin L (2014), sendo possível apreender a prática dos profissionais. Optou-se por usar as abreviaturas da nomenclatura "Entrevistado" e enumerá-los sucessivamente, com o firme propósito de preservar a identidade civil dos sujeitos de pesquisa.

A partir da decomposição dos textos em blocos organizados e leitura exaustiva dos mesmos, foi possível a identificação de categorias de análise, seguindo da inferência dos resultados e chegando à fase de interpretação dos resultados e indo além do material coletado, sendo adotados referenciais teóricos próprios do ensino em saúde, bem como da especificidade da área da saúde mental.

O protocolo de pesquisa foi submetido e aprovado no Comitê de Ética em Pesquisa da Universidade Federal de Alagoas, sob a CAAE 45167618800005013.

\section{RESULTADOS E DISCUSSÃO}

Os resultados são apresentados em quadros, seguidos das respectivas discussões. Destacamos o perfil dos docentes em relação ao sexo, faixa etária, área de graduação, se divide a disciplina/módulo/conteúdos com outros docentes (Quadro 1).

Quadro 1 - Caracterização dos docentes de saúde mental de universidades públicas do estado de Alagoas, 2016.

\begin{tabular}{|c|c|c|c|c|}
\hline Sujeito & Sexo & Faixa etária & Área de graduação & Divide a disciplina \\
\hline Ent.01 & $\mathrm{M}$ & 45 & Medicina & Sim \\
\hline Ent.02 & $\mathrm{F}$ & 43 & Enfermagem & Sim \\
\hline Ent.03 & $\mathrm{F}$ & 42 & Terapia ocupacional & Não \\
\hline Ent.04 & $\mathrm{F}$ & 40 & Enfermagem & Sim \\
\hline Ent.05 & $\mathrm{M}$ & 40 & Medicina & Sim \\
\hline Ent.06 & $\mathrm{F}$ & 34 & Medicina & Sim \\
\hline Ent.07 & $\mathrm{M}$ & 44 & Psicologia & Sim \\
\hline Ent.08 & $\mathrm{F}$ & 40 & Psicologia & Sim \\
\hline Ent.09 & $\mathrm{F}$ & 45 & Serviço Social & Não \\
\hline Ent.10 & $\mathrm{M}$ & 42 & Psicologia & Sim \\
\hline Ent.11 & $\mathrm{F}$ & 38 & Enfermagem & Sim \\
\hline
\end{tabular}

Fonte: Matias EC, et al., 2020. 
A partir das informações apresentadas percebe-se a heterogeneidade etária, variando de 34 a 45 anos, com predominância feminina, consonante com a constituição de gênero das categorias profissionais estudadas. Há compartilhamento das disciplinas e/ou módulos que abordam a saúde mental. Seria interessante uma relação mais interdisciplinar entre os cursos da mesma instituição de ensino, de modo a refletir a composição das equipes dos serviços de saúde.

A partir das falas dos docentes, foi possível destacar alguns temas que se referiam à prática educativa dos professores na perspectiva da Política de Saúde Mental vigente: 1) Variedade de recursos didáticos utilizados pelos docentes; 2) Utilização de conteúdos e referenciais de orientações distintas, quando não, antagônicas; 3) Formação discente com enfoque nos processos de humanização e valorização das necessidades de saúde dos usuários; 4) Campo psiquiátrico e campo psicossocial: diferenças nas abordagens e nos cenários de aprendizagem.

O aprofundamento nos principais temas evidenciou dissonâncias na prática educativa, no que se refere à finalidade a que se propõe o ensino em saúde mental, considerando a perspectiva psicossocial e antimanicomial. Assim sendo, a formação de profissionais críticos e reflexivos, quanto ao processo de Reforma Psiquiátrica e a utilização da Política Nacional de Saúde Mental e das DCNs, devem se fazer materializadas na prática docente. A partir da constituição das categorias de análise, chegou-se às seguintes categorias empíricas e subcategorias:

\section{Campo psiquiátrico e campo psicossocial: diferenças nas abordagens e nos cenários de aprendizagem}

Esta categoria se refere às proximidades existentes entre o ensino em saúde mental e as diferentes abordagens na área, evidenciando a amplitude do campo e organização dos docentes, em relação ao processo de ensino-aprendizagem, a partir do mesmo. Variedade de ferramentas, estratégias didáticas e cenários para a articulação teórico-prática utilizados pelos docentes.

A Política Nacional de Saúde Mental, ainda em vigor, circunscreve os dispositivos da RAPS prioritários para o cuidado aos usuários que tem necessidades de saúde mental, sendo o CAPS, o serviço mais presente na articulação entre ensino e serviços, seguido pelo Consultório na Rua, o que têm possibilitado avanços nas práticas de ensino. Podem-se observar os cenários de aprendizagem e de articulação teórico-prática selecionados para formação em saúde mental (Quadro 2). 


\section{Atacervo+}

Revista Eletrônica Acervo Saúde | ISSN 2178-2091

Quadro 2 - Apresentação dos cenários utilizados na articulação teórico-prática, das estratégias didáticas e das ferramentas/recursos utilizados pelos docentes, Maceió, 2016.

\begin{tabular}{|c|c|c|c|c|c|c|c|c|c|c|c|c|}
\hline . & & & & & colha & entes & & & & & & \\
\hline & Hospital psiquiátrico & $\mathrm{X}$ & $\mathrm{X}$ & $\mathrm{x}$ & $\mathrm{x}$ & & & & & & & \\
\hline & Centro de Atenção Psicossocial & $x$ & $x$ & $x$ & $x$ & $x$ & $x$ & $x$ & $x$ & $\mathrm{X}$ & & \\
\hline & Clínica de internação involuntária & & & & & & & & & & & \\
\hline & Comunidade terapêutica/ acolhedora & $\mathrm{x}$ & & & & & & & & & & \\
\hline & Unidade básica de saúde & $\mathrm{X}$ & $\mathrm{x}$ & & & & & & & & & \\
\hline$\cdot \frac{\sqrt{\pi}}{\pi}$ & Ambulatório de saúde mental & $\mathrm{x}$ & $\mathrm{x}$ & $\mathrm{x}$ & $\mathrm{x}$ & & & & & & & \\
\hline & Consultório na rua & $\mathrm{x}$ & $\mathrm{x}$ & $\mathrm{x}$ & $\mathrm{X}$ & $\mathrm{X}$ & & & & & & \\
\hline & Outro & 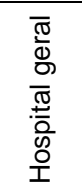 & \) & 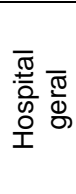 & 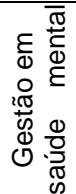 & 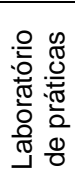 & & & & & & \\
\hline & Aula expositiva & $x$ & $x$ & $\mathrm{x}$ & $\mathrm{x}$ & & & & & & & \\
\hline & Discussão em pequenos grupos & $x$ & $x$ & $\mathrm{x}$ & $\mathrm{x}$ & $\mathrm{x}$ & $\mathrm{x}$ & $\mathrm{x}$ & $\mathrm{x}$ & $\mathrm{x}$ & & \\
\hline$\stackrel{\circlearrowright}{=}$ & Problematização & $x$ & $x$ & $\mathrm{x}$ & $\mathrm{x}$ & $\mathrm{x}$ & $\mathrm{x}$ & $\mathrm{x}$ & $\mathrm{x}$ & & & \\
\hline 흥 & Aprendizagem baseada em problemas & $x$ & $x$ & $x$ & & & & & & & & \\
\hline 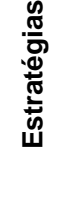 & Outro & 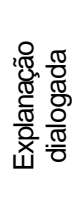 & 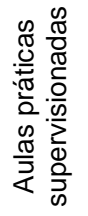 & $\begin{array}{l}\frac{\widehat{d}}{\frac{0}{\alpha}} \\
\frac{\dot{\phi}}{0} \\
\mathbb{\Upsilon}\end{array}$ & 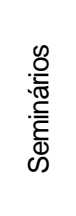 & 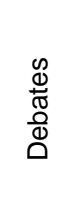 & 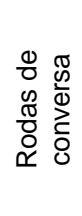 & 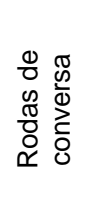 & 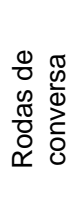 & & & \\
\hline & Projetor de multimídia & $x$ & $x$ & $x$ & $x$ & $x$ & $x$ & $x$ & $x$ & $x$ & $x$ & $\mathrm{x}$ \\
\hline & Vídeo sobre o tema & $\mathrm{X}$ & $\mathrm{X}$ & $\mathrm{X}$ & $\mathrm{X}$ & $\mathrm{X}$ & $\mathrm{X}$ & $\mathrm{X}$ & $\mathrm{X}$ & $\mathrm{X}$ & $\mathrm{X}$ & \\
\hline$\stackrel{0}{\frac{\omega}{z}}$ & Material de papelaria & $\mathrm{X}$ & $\mathrm{x}$ & $\mathrm{x}$ & $\mathrm{x}$ & & & & & & & \\
\hline$\Phi$ & Mídias digitais (facebook, twitter, Whatsapp, etc) & $\mathrm{X}$ & $\mathrm{x}$ & $\mathrm{x}$ & $\mathrm{X}$ & $\mathrm{X}$ & $\mathrm{X}$ & $\mathrm{X}$ & & & & \\
\hline 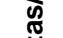 & Música & $\mathrm{X}$ & $\mathrm{X}$ & $\mathrm{X}$ & $\mathrm{X}$ & $\mathrm{X}$ & $\mathrm{X}$ & & & & & \\
\hline$\frac{\tilde{c}}{\sigma}$ & Texto & $\mathrm{X}$ & $\mathrm{X}$ & $\mathrm{X}$ & $\mathrm{X}$ & $\mathrm{X}$ & $\mathrm{X}$ & $\mathrm{X}$ & $\mathrm{X}$ & $\mathrm{X}$ & & \\
\hline Ӗ & Dramatização & $X$ & $\mathrm{X}$ & $\mathrm{x}$ & $\mathrm{x}$ & $\mathrm{x}$ & & & & & & \\
\hline 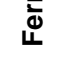 & Outro & 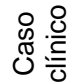 & & & & & & & & & & \\
\hline
\end{tabular}

Fonte: Matias EC, et al., 2020. 
Esses espaços assumem o lugar do diálogo entre trabalho e educação; portanto, neles devem ser produzidos movimentos na direção de transformações dos tradicionais modelos de ensino para as formações na saúde, estes que se mostram com dificuldades para responder adequadamente às necessidades da população (HENRIQUES RLM, 2005).

Os CAPS são estratégicos na construção e implementação de novas práticas de cuidado voltadas às pessoas que têm transtorno mental ou aquelas que têm problemas relacionados ao uso abusivo de álcool e outras drogas. Esses serviços utilizam-se de ferramentas como os projetos terapêuticos singulares e os conceitos da Clínica ampliada (BRASIL, 2009). Já os Consultórios na Rua garantem cuidado às pessoas que estão em situação de vulnerabilidade social, no contexto de rua, fazendo parte do Plano integrado de enfrentamento ao crack e outras drogas (BRASIL, 2011).

Muito tem-se discutido, sobre a eficácia desses serviços e a necessidade real de seu acesso. Em Alagoas temos, equivalente as Comunidades Terapêuticas, as chamadas Comunidades Acolhedoras, serviços que até então não dispostos na RAPS e, consequentemente, na política de saúde mental, mas que atendem aos usuários de álcool e outras drogas em situação de vulnerabilidade, sendo apontadas por somente um dos interlocutores.

Albuquerque VC, et al. (2008), expõem que "os espaços onde se dá o diálogo entre o trabalho e a educação assumem lugar privilegiado para a percepção que o estudante vai desenvolvendo acerca do outro no cotidiano do cuidado", onde ocorre interação entre os diversos atores e que são legítimos espaços de cidadania. Os entrevistados, ao elegerem serviços substitutivos enquanto locais de articulação, favorecem um ensino pautado às mudanças observadas, desde a década de 1970, para a oferta do cuidado em saúde mental.

Para Albuquerque VS e Campos JCL (2010), ao diversificar esses espaços e as estratégias de ensino, valorizam o protagonismo e a autonomia dos discentes e que o cotidiano da Reforma Psiquiátrica brasileira vem sofrendo mudanças e as possibilidades oferecidas aos usuários estão presentes na formação dos futuros profissionais e isso requer a elegibilidade de cenários para articulações do ensino com a atenção desenvolvida.

Autores como Amarante P (2007), Lancetti A (2006) e Hirdes A (2009), afirmam que a rede de atenção psicossocial é constituída por muitos serviços e que, na experiência da formação em saúde, faz-se imperativo vivenciar essa rede, pois é nesse cenário que se encontrará o espaço para desmedicalizar e desinstitucionalizar.

As estratégias utilizadas pelos docentes, supracitadas, foram elencadas algumas pedagogias geralmente utilizadas. Percebe-se que os entrevistados utilizam uma variedade dessas estratégias, demonstrando ainda que várias são as abordagens ativas dentro do processo, em contramão às abordagens tradicionais no ato de ensinar/aprender.

Essas perspectivas favorecem a autonomia e o processo crítico-reflexivo dos sujeitos, em detrimento de um movimento pedagógico conservador e distante dos contextos locais. Na atualidade, tem ocorrido discussão sobre a necessidade das instituições de ensino reconstruírem seu papel social, aproximando-se da realidade vivenciada pelos sujeitos, nesse caso, o cotidiano onde se dão as práticas pedagógicas (MITRE SM, et al., 2008).

A discussão em pequenos grupos (DPG) é a metodologia utilizada por nove dos onze docentes e, de acordo com Ruiz-Moreno L (2004), na DPG, o docente assume o lugar de tutor e orienta a discussão a partir de diferentes perspectivas, estimulando que o discente traga para o lócus, conhecimentos prévios e experiências. Essa proposta estimula a aprendizagem significativa com grupos de aproximadamente 10 alunos.

Defendida por Freire P (2014), dentre as várias correntes epistemológicas baseadas em teorias críticas, tem-se confirmado no meio educacional de nosso País, a "pedagogia libertadora" ou "pedagogia da problematização". Seguindo a linha freireana, pensar o tema da educação como um ato de afirmar a liberdade é incorporar a essa discussão o modo como esse pensamento foi instaurado historicamente, como também reconhecer o fato da opressão, do mesmo modo que há luta pela libertação. 
Percebe-se que os docentes têm se debruçado em seus espaços de ensino com uma ampliada caixa de ferramentas, o que pode traduzir uma abordagem que assimile o conhecimento a partir dos seguintes estilos de aprendizagem (FLEMING ND, 2001): visual/leitura e escrita (vídeos, mídias, textos, etc), cinestesia (dramatização) e audição (músicas), e que há um respeito à forma como cada um aprende e apreende as informações a partir das estratégias cognitivas utilizadas.

Dentre os autores que discutem as ferramentas de ensino, Mazzioni S (2013) o faz a partir de outras denominações como: recursos, instrumentos, etc. Nessa perspectiva, além de questões que interferem no referido processo, as ferramentas de ensino devem ter o objetivo de motivação, envolvendo os discentes no contexto.

Destacamos que a totalidade dos docentes indicou utilizar mais de um recurso didático, sendo o projetor de multimídia utilizado por todos, seguido de vídeos sobre a temática e textos. Para Albuquerque VC e Campos JCL (2010), há uma opção curricular em utilizar filmes/vídeos como recurso educacional, pois, o cinema é um instrumento de educação.

No que se refere à utilização de tecnologia audiovisual, Amarante $\mathrm{P}$ e Rangel M (2009) referem que uma importante quantidade de vídeos tem demonstrado formas de superação do modelo psiquiátrico clássico. Esses recursos servem como imagens-objetivo do cuidado, bem como das possibilidades de construção de novos caminhos e superações de desafios do cotidiano na área.

Observa-se, ainda, a utilização no cotidiano dos docentes das Tecnologias da Informação e Comunicação (TIC), sendo as mesmas definidas por Torres AAL, et al. (2015) como "ferramentas utilizadas para acessar, reunir, manipular, apresentar ou comunicar informações". Há uma tendência em se potencializar a discussão sobre inovação do ensino ao destacar a importância da aplicação das TIC.

Em relação às TIC, conforme Rezende F (2002), estão a possibilidade de interatividade, de interação à distância e de armazenamento e organização em sistemas multimídia das mais variadas.

Desse modo, tendo a disposição esse leque de recursos e tecnologias, o docente poderá selecionar e utilizar conforme os objetivos propostos, juntamente com planejamento de ensino e desenvolvimento de habilidades na docência em saúde.

\section{Utilização de conteúdos e referenciais de orientações distintas ou até antagônicas}

As políticas de saúde mental e as DCN para os cursos da saúde, têm exigido profissionais que incorporem em seu cotidiano conceitos ampliados e práticas de cuidado, além de uma prática dialogada com a teoria.

Quanto aos conteúdos, um dos interlocutores considera importante abordar os transtornos psiquiátricos em geral. Outro entrevistado inclui nos conteúdos as urgências psiquiátricas, risco de suicídio, intoxicação e abstinência de álcool e outras drogas ou psicotrópicos, ataque de pânico e delírio.

Uma das docentes refere que, além dos transtornos, o relacionamento terapêutico, a política de saúde mental, a abordagem familiar, seguindo um modelo de Genograma e Ecomapa e o Projeto Terapêutico Singular (PTS), são imprescindíveis para o ensino-aprendizagem.

Para docentes de Psicologia, o ensino tem se dado a partir da discussão sobre o conceito de doença, de saúde e de loucura, pois, segundo um deles, é a temática onde ele tem sentido maior necessidade de discutir. Ao tempo em que o docente também refere que há uma tendência a minimizar, a discussão apenas aos aspectos políticos na área, não enfatizando os aspectos epistemológicos, discussões sobre psicopatologia.

[...] pensar na saúde mental como área de atuação, exige uma metodologia específica, uma reflexão política em relação a essa área porque eu tenho uma impressão que na psicologia há uma tendência atual em querer reduzir a epistemologia política (Ent. 10).

Os sujeitos abordam a importância de dialogar com o conceito de saúde da OMS, mas relacionado à "clínica dos sujeitos". Percebe-se um ponto em que as formas de pensar Clínica e Política não sigam os mesmos direcionamentos, a depender dos referenciais adotados. 
[...] trabalhar uma clínica do sujeito e que tem tudo a ver com clínica ampliada, essa discussão que vem aí de construção de projetos terapêuticos singulares, é central. $O$ segundo eixo é pensar uma clínica articulada o tempo inteiro com a Política, e quando eu falo Política, é trazendo a questão da Polis, uma clínica que vai pensar os jeitos de ser e de viver nas cidades, nos nossos territórios, nos locais em que a gente vive (Ent. 07).

Os conteúdos trabalhados no curso de Serviço Social estão voltados à Reforma Psiquiátrica e ao Movimento de Luta Antimanicomial, assim como o perfil dos usuários dos serviços de saúde mental, procurando entender a situação econômica e social, além da organização política.

[...] sentimos a necessidade de diferenciar a Reforma Psiquiátrica da Luta Antimanicomial, que foi uma coisa que aprendemos com o tempo, porque às vezes parecia que era a mesma coisa, mas depois começamos a estudar e fizemos questão de trabalhar isso, até por que existem diferentes perspectivas de se entender e se fazer a Reforma (Ent. 09).

Corroborando com estudo de Villela JC, et al. (2013), ao investigar o ensino de saúde mental, foram achados conteúdos referentes à história da psiquiatria, políticas de saúde, transtornos mentais, rede de apoio, dentre outros. Para Luckesi CC (2007), a educação se formatará enquanto transformadora da sociedade, incorporando pressupostos e conceitos para fundamentar os seus caminhos, visando transformar ideias préconcebidas e pré-existentes, para reconstruí-las.

Os conceitos e conteúdos que fundamentam o ensino nas duas instituições estudadas nos faz pensar que o escopo utilizado transcende ao ensino da Psiquiatria Clássica, buscando um ensino mais problematizador.

Se tratamos de uma área com legislação e política específica, deve estar em consonância com o que está posto, sendo fruto de intenso debate técnico e político, inclusive com as recentes proposta (BRASIL, 2019), do governo federal, de retorno dos incentivos aos hospitais psiquiátricos e comunidades terapêuticas de cunho religioso.

A seleção do conteúdo programático é importante e, para que a educação se caracterize como transformadora da sociedade, necessita de pressupostos e conceitos que fundamentem e orientem os seus caminhos.

Para Villela JC, et al. (2013), o currículo que privilegia o ensino centrado na doença e que predominou (e ainda predomina) no mundo, é um modo de conceber a saúde mental, e todo sofrimento psíquico é classificado e "enquadrado" nos diagnósticos e num modo de existir meramente biológico e onde o sujeito é tratado em ambientes de isolamento, com características manicomiais.

A Política Nacional de Saúde Mental, tendo como base a Reforma Psiquiátrica brasileira, é um terreno de disputa política, estando o ensino também em disputa. A psicopatologia, amplamente ensinada nas escolas médicas, é, segundo Silveira RD (2013), fundamental em termos de ensino.

Ao caracterizar a formação dos alienistas, os autores referem que "o modelo de formação dos alienistas seguia a mesma tradição da clínica médica constituída entre os séculos XVIII e XIX: acompanhar o mestre, segui-lo, adotar seu estilo, copiá-lo à exaustão. Aprender a detectar os mínimos sinais, conceituá-los e classificá-los, era o que se esperava de um estudante, ao ver essa tarefa cumprida, pensava que estava fazendo clínica a partir dessa prática" (SILVEIRA RD, 2013).

No que tange aos pressupostos teóricos e práticos utilizados, os docentes têm optado por referenciais bem diferentes, porém, apesar de formações diversas, utilizam premissas alusivas do processo de desinstitucionalização e Reforma Psiquiátrica no Brasil e no mundo. Outros entrevistados utilizam da Política de Saúde Mental e dos documentos ministeriais sobre práticas de Acolhimento, Humanização e Clínica Ampliada. Já os sujeitos com formação médica, enveredam, majoritariamente, por abordagens ligadas aos referenciais biomédicos, conforme a seguinte fala:

Eu particularmente sigo muito os artigos e publicações mais novas sobre a dependência química, esse envolvimento do crack, tudo o que é atividade que é feita pelo Laranjeira, Ribeiro, o velho Kaplan ainda é muito utilizado, o DSM V[...] (Ent. 01). 
Outros autores utilizados são: Kapczinski e Quevedo, que tratam sobre emergências psiquiátricas e os manuais diagnósticos DSM IV e CID 10. Para Semiologia, utilizam autores como Sadock, Stahl, Pondé, Reis e Dalgalarrondo.

Para docentes com formação não médica, pensadores importantes para o debate seriam Foucault, Canguillhem, Freud, Jung, Pigeau, Lapassade, Bleger, Benevides, Barros de Barros, Passos, Mary Jane Spink, Paim e Campos.

Além dos autores tradicionais e contemporâneos da Psiquiatria e da Reforma Psiquiátrica, estudiosos da formação em saúde foram citados como necessários à formação e a própria base de constituição do ensinoaprendizagem, sendo eles: Ceccim, Feuerwerker e Freire. O referencial da Pós-modernidade que traz a transformação dos sujeitos e das ciências também é utilizado, fazendo-se referência a Edgar Morin.

\section{CONCLUSÃO}

O campo da Saúde Mental e da Atenção Psicossocial apresenta-se em processo de múltiplas transformações, seja na atenção prestada aos sujeitos em sofrimento psíquico, seja na formação dos profissionais, tendo as práticas docentes pautadas, em sua maioria, pela Reforma Psiquiátrica. Nesta pesquisa, os docentes têm se debruçado sobre um ensino construído a partir da psiquiatria contemporânea, até aqueles com visões reformistas. Percebem-se aproximações e distanciamentos quanto aos espaços de cuidado para a prática do ensino, desde uma lógica territorial até aqueles de lógica institucionalizante. Fazse necessário considerar as limitações desse estudo, tendo o mesmo não aprofundado a trajetória com que cada curso de graduação construiu o currículo e a inserção da saúde mental nesse percurso. Identificou-se as metodologias ativas presentes no do ensino em saúde mental e que há acesso às variadas ferramentas de ensino, considerando-se, de fundamental importância para se respeitar as formas como cada um constrói o conhecimento.

\section{REFERÊNCIAS}

1. ALBUQUERQUE VS, CAMPOS JCL. Saúde mental no currículo integrado do Curso de Enfermagem do Unifeso: diversificação de cenários e de estratégias de ensino-aprendizagem. R. pesq.: cuid. fundam. online (Rio de Janeiro). 2010; 2(4): p. 1516-1527.

2. ALBUQUERQUE VS, et al. A Integração ensino-serviço no contexto dos processos de mudança na formação superior dos profissionais da saúde. Revista Brasileira de Educação Médica (Rio de Janeiro). 2008; 32 (3): p. 356 - 362.

3. AMARANTE P, RANGEL M. A liberdade é terapêutica: reinventando vida na Reforma Psiquiátrica. R. Elet. de Com. Inf. Inov. Saúde (Rio de janeiro). 2009; 3(4): p. 10-16.

4. AMARANTE P. Saúde Mental e Atenção Psicossocial. Rio de Janeiro: Fiocruz; 2007.

5. BARDIN L. Análise de conteúdo. São Paulo: Edições 70; 2014.

6. BATISTA NA, BATISTA SH, organizadores. A docência em saúde: desafios e perspectivas. São Paulo: Senac; 2004. p. 18-31.

7. BOTTI SHO. O Papel do Preceptor na Formação de Médicos Residentes: um estudo de residências em especialidades clínicas de um hospital de ensino. [tese]. Rio de Janeiro (RJ): Escola Nacional de Saúde Pública Sergio Arouca; 2009.

8. BRASIL. Congresso Nacional. Lei no 10.172, de 9 de Janeiro de 2001. Aprova o Plano Nacional de Educação (20012010) - PNE e dá outras providências. Diário Oficial da União. 4 Maio 2001 a.

9. BRASIL. Ministério da Educação. Conselho Nacional de Educação. Câmara da Educação Superior. Parecer CNE/CES № 1.133, de 7 de Agosto de 2001. Estabelece as diretrizes curriculares para os cursos de ciências biológicas. Diário Oficial da União. 3 Out $2001 b$.

10. BRASIL. Ministério da Saúde (BR). Secretaria de Atenção à Saúde. Política Nacional de Humanização da Atenção e Gestão do SUS.. Clínica ampliada e compartilhada. Brasília (DF): MS; 2009. (Série B. Textos Básicos de Saúde).

11. BRASIL. Ministério da Saúde. Secretaria de Atenção à Saúde. Departamento de Ações Programáticas Estratégicas. Coordenação-Geral de Saúde Mental, Álcool e Outras Drogas. NOTA TÉCNICA № 11/2019CGMAD/DAPES/SAS/MS. Assunto: Esclarecimentos sobre as mudanças na Política Nacional de Saúde Mental e nas Diretrizes da Política Nacional sobre Drogas. Brasília, 2019.

12. BRASIL. Portaria $n^{\circ} 122$, de 25 de janeiro de 2011. Define as diretrizes de organização e funcionamento das Equipes de Consultório na Rua. Diário Oficial da União, 26 jan 2012.

13. BRASIL. Portaria $n^{\circ} 336$, de 19 de fevereiro de 2002. Define e estabelece diretrizes para o funcionamento dos Centros de Atenção Psicossocial. Brasília, DF: Ministério da Saúde; 2002.

14. BRITO FILHO GT, et al. A docência no cotidiano da sala de aula universitária. Conceitos (João Pessoa). $2004 ; 1$ (1): $14-18$. 
15. CARNEIRO LA, PORTO CC. Saúde mental nos cursos de graduação: interfaces com as Diretrizes Curriculares nacionais e com a Reforma Psiquiátrica. Cadernos Brasileiros de Saúde Mental (Florianópolis). $2014 ; 6$ (14): p.150167

16. FLEMING ND. Teaching and learning styles: VARK strategies. New Zealand: Christchurch; 2001.

17. FREIRE P. Educação como prática de liberdade. Rio de Janeiro: Paz e Terra; 2014.

18. HENRIQUES RLM. Interlocução entre ensino e serviço: possibilidades de ressignificação do trabalho em equipe na perspectiva da construção social da demanda. In: Pinheiro R, Mattos RA, organizadores. Construção social da demanda. Rio de Janeiro: IMS-UERJ/CEPESC/ABRASCO; 2005. p.147-159.

19. HIRDES A. A reforma psiquiátrica no Brasil: uma (re) visão. Ciência e Saúde Coletiva (Rio de Janeiro). 2009; 14(1): P. 297-305.

20. LANCETTI A. Clínica Peripatética. São Paulo: Hucitec; 2006.

21. LUCKESI CC. Filosofia da Educação. São Paulo: Cortez, 2007.

22. MAZZIONI S. As estratégias utilizadas no processo de ensino-aprendizagem: concepções de alunos e professores de ciências contábeis. Revista Eletrônica de Administração e Turismo - ReAT. 2013; 2 (1): p. 93-109.

23. MITRE SM et al. Metodologias ativas de ensino-aprendizagem na formação profissional em saúde: debates atuais. Ciência e Saúde Coletiva (Rio de Janeiro). 2008; 13 (Sup 2): p. 2133-2144.

24. REZENDE F. As novas tecnologias na prática pedagógica sob a perspectiva construtivista. Ensaio - Pesquisa em Educação em Ciências (Belo Horizonte). 2002; 2 (1): p.1-18.

25. RIBEIRO MC. A saúde mental em Alagoas: trajetória da construção de um novo cuidado. Maceió: Grafpel; 2014.

26. RISTOW AM. A formação humanística do médico na sociedade do século XXI: uma análise curricular. [dissertação]. Curitiba (PR): Universidade Federal do Paraná; 2007.

27. RUIZ-MORENO L. Trabalho em grupo: experiências inovadoras na área da educação em saúde. In: Batista NA, Batista SH, organizadores. Docência em saúde: temas e experiências. São Paulo: Senac; 2004. p. 85-99.

28. SILVEIRA RD. Psicopatologia e ensino em Saúde Mental. In: Lobosque AM, Silva CR, organizadores. Saúde Mental: marcos conceituais e campos de prática. Belo Horizonte: CRP 04; 2013. p. 47-55.

29. TORRES AAL, et al. Uso de tecnologias de informação e comunicação no ensino na saúde: revisão sistemática 2010 2015. Revista Eletrônica Gestão \& Saúde (Brasília). 2015; 6 (2): p. 1883-1889.

30. VILLELA JC, et al. O ensino de saúde mental na graduação de Enfermagem na perspectiva do estudante. Texto Contexto Enferm (Florianópolis). 2013; 22(2): p. 397-406.

31. YASUI S. Rupturas e encontros: desafios da Reforma Psiquiátrica brasileira. Rio de Janeiro: Editora Fiocruz, 2010. Coleção Loucura e Cidadania. 